\title{
A Pipeline Abnormal Signal Detection Method Based on 1D-Faster R-CNN
}

\author{
Zhen Zhang a and Weiguo Lin ${ }^{\mathrm{a}, 1}$ \\ ${ }^{a}$ College of Information Science and Technology, Beijing University of Chemical \\ Technology, Beijing 100029, China
}

\begin{abstract}
Aimed at the detection difficulty of local abnormal signals during pipeline operation, this paper takes the local abnormal signals as the detected targets, and proposes a new method based on target detection to extract abnormal signals with different amplitude and shape; and for the case where there are few actual leak samples, combined with the characteristics that the training samples of each module of the model itself are derivative samples of the original sample, so as to realize the small sample training of the model. Finally, a new pipeline leak detection and location method is proposed by combining the 1D-faster R-CNN with the cross-correlation location method based on signal matching. The experimental results show that the proposed method effectively extracts local abnormal signals, accurately alarms leak signals, and eliminates the false alarms caused by the recognition errors of normal signals.
\end{abstract}

Keywords. target detection, local abnormal signal extraction, small sample, pipeline leak detection

\section{Introduction}

Pipeline is an important infrastructure in petroleum transportation. Due to pipeline aging, corrosion and third-party damage, pipeline leak accidents often occur [1-3], which can lead to serious safety accidents [4], especially for the transportation of some dangerous substances, such as liquid chlorine and hydrogen. Therefore, the monitoring of pipeline leak is critical, for which domestic and foreign scholars have conducted a large number of studies [5-8].

In recent years, deep learning has been widely used in image processing, natural language processing and other fields [9-10]. For pipeline leak detection, Kang et al. [11] proposed one-dimensional convolutional neural network and support vector machine (1D-CNN-SVM) model to classify leak and normal signals of water supply pipelines; Zhou et al. [12] proposed a classification model based on improved splinelocal mean decomposition (ISLMD) and CNN, and identified leak signals with different leak apertures. Wang et al. [13] utilized time domain statistical features of normal samples to establish a diagnosis model based on support vector data description (SVDD) and implemented pipeline leak diagnosis. But it cannot extract the local abnormal information of an abnormal signal, and is hard to avoid false and missing alarm after long continuous running.

${ }^{1}$ Corresponding Author: Weiguo Lin, College of Information Science and Technology, Beijing University of Chemical Technology, Beijing 100029, China ; Email: linwg@mail.buct.edu.cn 
And existing pipeline leak detection methods take a whole signal as the detection object, and fail to provide its local abnormal information, such as the number of local abnormal signals and their positions in a whole signal. However, in practical applications, the local abnormal information is vital to accurately locate and eliminate false and missing alarms. Wang et al. [14] proposed a model-free abnormal signal separation method, which realized the detection of local abnormal signals. However, it is based on the signal-to-noise ratio (SNR) increment of interval sub-signals, which is likely to cause the missing detection of relatively obvious abnormal signals.

In image processing, the target detection method can give the specific location of the target in the image and is suitable for multi-scale object detection. Girshick et al. proposed region-based convolutional neural networks (R-CNN) [15] and Fast R-CNN [16] successively, which greatly improved the accuracy of image target recognition. Subsequently, Ren et al. [17] proposed Faster R-CNN, making progress again in detection speed and identification accuracy. In addition, since the training samples of each module in the Faster R-CNN model are derivative samples of the original samples, and the number of which is much larger than original samples, it is very suitable for model training under the condition of small samples. Based on the faster detection speed, accurate target recognition, and small sample modeling of Faster R-CNN, and combined with the characteristics of fewer pipeline leak samples, this paper proposes an abnormal signal detection method based on 1D-Faster R-CNN. This method takes leak signals and interfering signals as training samples to establish a local abnormal signal extraction model, which reduces the dependence of model training on the number of samples. The position and number of local abnormal signals detected quickly and accurately are very beneficial for reducing false and missing alarms, and improving the accuracy of leak location.

The rest of the paper is organized as follows. Section 2 introduces the abnormal signal extraction methods based on 1D-Faster R-CNN from five aspects: data preprocessing, anchor point and boundary interval regression for one-dimensional signals, model structure, derivative samples and model training. Experimental results are presented in Section 3. And this paper is summarized and concluded in Section 4.

\section{Local abnormal signal detection method based on 1D-Faster R-CNN}

\subsection{Data preprocessing}

Deep neural network usually needs to normalize data, and commonly used data normalization methods are maximum minimum normalization and $z$-score standardization. And z-score standardization is more sensitive to outliers. According to the purpose of extracting abnormal signals, this paper uses z-score standardization to preprocess the data. The calculation of $\mathrm{z}$-score standardization is:

$$
x^{*}=\frac{x-\bar{x}}{\sigma}
$$

Where $x$ is the signal amplitude, $\bar{x}$ is the signal mean, $\sigma$ is the standard deviation of a signal, and $x^{*}$ is the signal amplitude after $\mathrm{z}$-score standardization.

Since the actual leak samples are difficult to obtain and deep learning requires a large number of samples, samples need to be expanded. In this paper, each abnormal 
signal is regarded as an independent target, so the time sequence of the abnormal signal does not affect its detection. Based on this, this paper uses Eq. (2) to carry out symmetric transformation of the input sample to realize the expansion of the training dataset. That is, the signal is symmetrically flipped along the axis of symmetry to double the size of the dataset.

$$
\left\{\begin{array}{l}
x_{\text {length-i }}=x_{i} \\
1 \leq i \leq \text { length }
\end{array}\right.
$$

Where length is the length of the signal, and $x$ is the amplitude of the signal.

Generally, infrasound signals of pipeline need to be filtered to improve the SNR. However, the boundary effect of filtering sometimes brings serious outliers at the signal boundary, which affects the detection of local abnormal signals. Additionally, the filtered signal becomes smoother, which affects the convergence of the model and easily lead to the overfitting of the model. Therefore, the original signal is not filtered in this paper.

\subsection{Anchor, candidate interval and boundary interval regression}

In one-dimensional signals, the position of each feature point extracted from the original signal is called anchor, and each anchor is associated with $\mathrm{k}$ candidate intervals of different sizes and scales. Referring to the method of literature [17], this paper proposes a candidate interval generation method for one-dimensional signals, and utilizes Eq. (3) to generate 9 candidate intervals at each anchor point.

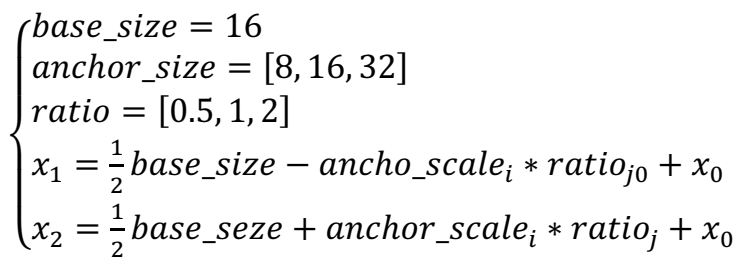

Where base_size is the base size of the candidate interval, anchor_size is the additional amount above the base size, ratio is the scale of anchor_size, $x_{1}$ and $x_{2}$ are the starting and end coordinate of the candidate interval, $j 0$ is the fixed subscript calculated each iteration, and $x_{0}$ is the anchor coordinate.

When the anchor coordinate is 0 , for example, the 9 candidate intervals generated at the anchor are $[4,12],[4,16],[4,24],[0,16],[0,24],[0,40],[-8,24],[-8,40],[-8$, $40],[-8,72]$. All anchor coordinates of a signal are $[0,16,32,48 \ldots, 2976,2992]$. Hence, 1683 candidate intervals can be generated from a signal. Figure 1 shows the position relationship between the 9 candidate intervals associated with an anchor point and a local abnormal signal.

For boundary interval regression, this paper adopts two position transformation parameters [15]. The proposed and predicted intervals are $P=\left\{P_{x}, P_{w}\right\}$ and the $G=\left\{G_{x}, G_{w}\right\}$, where $P_{x}, P_{w}, G_{x}, G_{w}$ are the starting coordinates and interval widths of the proposed interval and predicted interval respectively. And the transformation from $P$ to $G$ is: 


$$
\left\{\begin{array}{l}
G_{x}=P_{x}+P_{w} * d_{x} \\
G_{w}=P_{w} * \exp \left(d_{w}\right)
\end{array}\right.
$$

And the position transformation parameter $d^{*}=\left\{d_{x}, d_{w}\right\}$ is:

$$
\left\{\begin{array}{l}
d_{x}=\left(G_{x}-P_{x}\right) / P_{w} \\
d_{w}=\log \left(G_{w} / P_{w}\right)
\end{array}\right.
$$

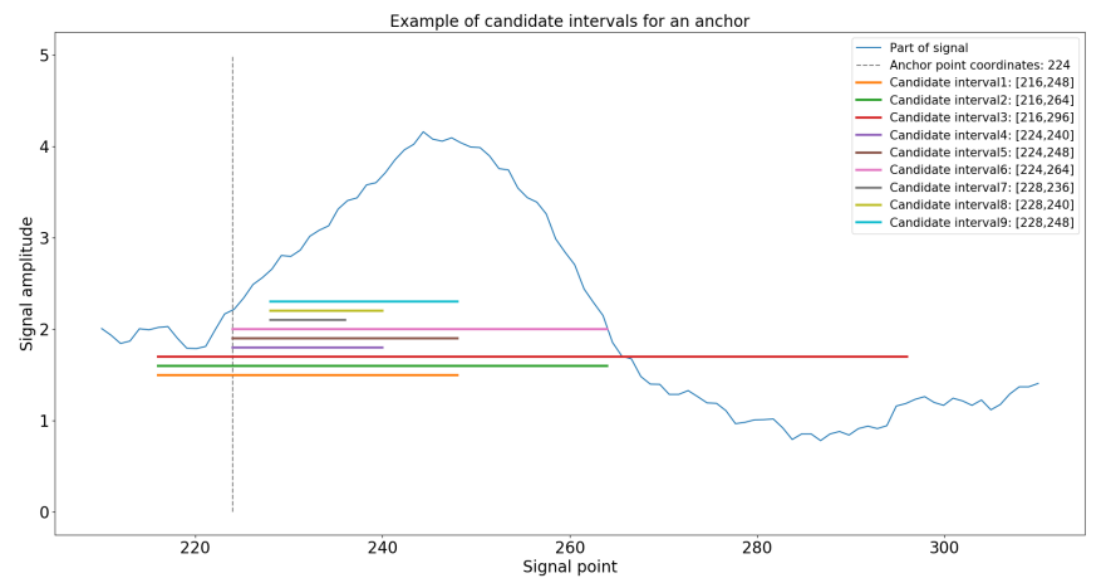

Figure 1. Example of candidate intervals for an anchor.

\subsection{Model structure}

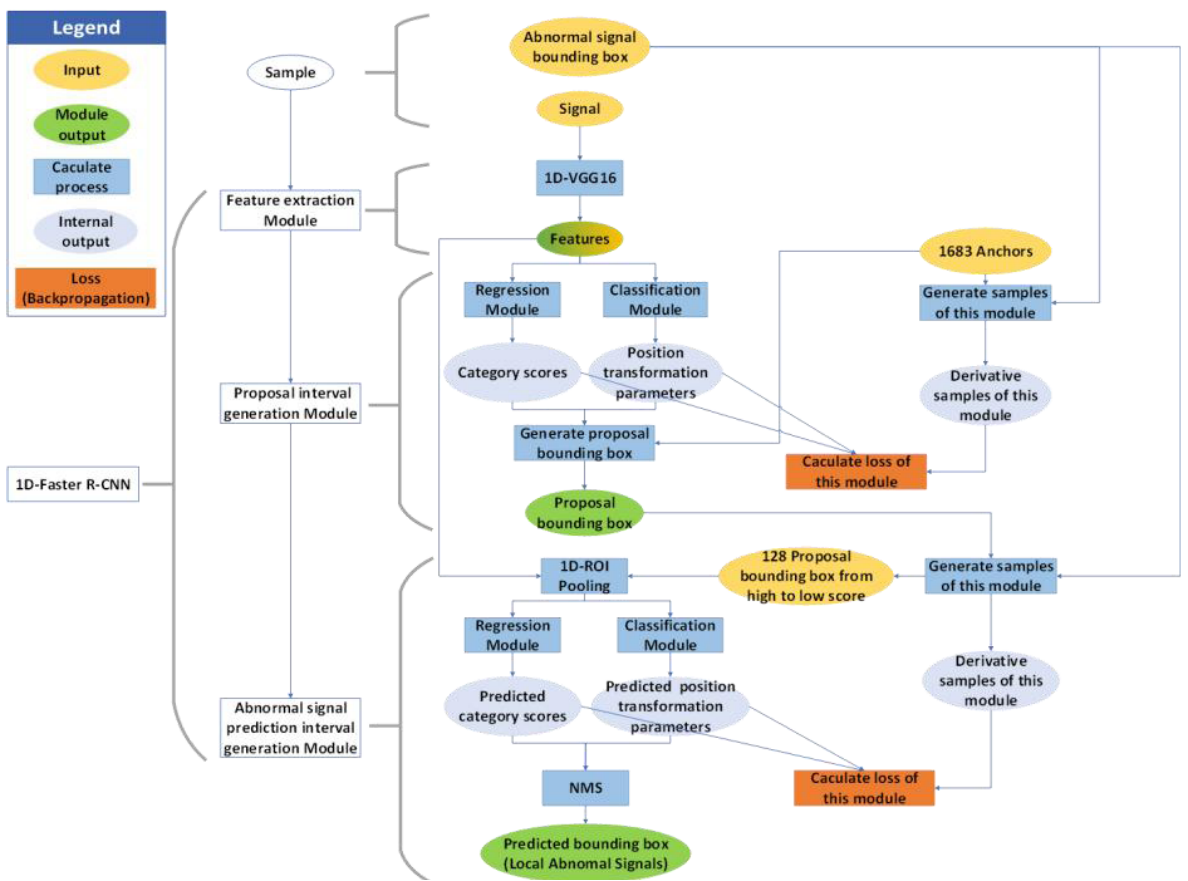

Figure 2. Model structure. 
1D-Faster R-CNN is composed of three modules: feature extraction module, proposal interval generation module, and abnormal signal prediction interval generation module. Figure 2 shows the overall structure of the model.

(1)The feature extraction module is used to extract the features of a signal, which can be realized with the full convolution calculation of visual geometry group network 16 (VGG16). In this paper, the length of a signal to be processed is 3000 points. And after the feature extraction module, the feature of 512 channels is obtained, and the number of feature elements in each channel is 187. Figure 3 shows some features extracted from normal and leak signals.
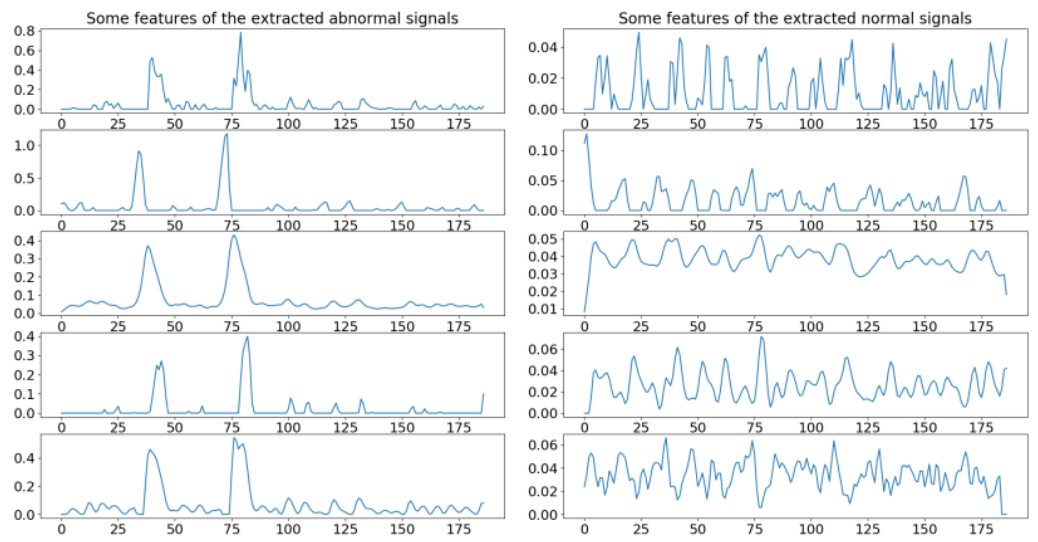

Figure 3. Some features extracted from normal and leak signals.

(2) The proposal interval generation module is to generate the proposal interval of the local abnormal signal to be detected. And it contains two models (classification and regression) and outputs the proposal intervals for abnormal signal identification. At the beginning of the module, a convolution layer is added to map the extracted features and further expand the feeling of the characteristic vector field. The receptive field size is 228. After the activation transformation (Rectified linear unit, ReLU), 512 feature vectors with length 187 are obtained, which are the input of the classification and regression models in this module.

The classification and the regression models are each composed of a convolution layer with a convolution kernel size of 1 , step size 1 , and 18 channels. They obtain the normal and abnormal signal category scores, and the two position transformation parameters of all candidate intervals, respectively. The position of each candidate interval is corrected according to the position transformation parameters obtained by regression. And the duplicate candidate intervals are deleted by non-maximum suppression (NMS) based on the abnormal signal category score of each candidate interval. When calculating NMS, the threshold of the intersection over union (IOU) is set to 0.7 . Finally, the 200 candidate intervals with the highest abnormal signal category scores are used as the final proposal intervals.

(3)Abnormal signal prediction interval generation module is to further realize the classification and interval position correction of local abnormal signal. It also includes classification and regression models. The 200 proposal intervals need further classification and position correction to obtain the final abnormal signal prediction interval. Firstly, each proposal interval is mapped to the feature extracted by feature extraction module to obtain the feature of corresponding interval. Then, by global 
maximum pooling, the features of the corresponding interval are divided into feature vector with the same length, and the obtained features are used as the input of the classification and regression models in this module.

The classification and regression models consist of a fully connected layer with 2 and 4 nodes, respectively. The classification and regression models obtain category scores and position transformation parameters of the normal and abnormal signal for all proposal intervals, respectively. Combining the results of the classification and regression models in this module, if there is an abnormal signal prediction interval, NMS is used to delete the repeated revised proposal interval to obtain the final abnormal signal prediction interval; if there is no abnormal signal prediction interval, the module has no output.

\subsection{Derivative samples}

The training samples are signal sequences marked with category labels and boundary intervals. The proposal interval generation module generates derivative samples for their own training through these samples and candidate intervals. Each sample is composed of position transformation parameters and category labels, and the number of derivative samples is much larger than training samples of the overall model. The process of generating derivative samples is:

(1) Compute the IOU between the elements in sets A and B, where A contains all the candidate intervals and the known boundary intervals of the signal sample, and $\mathrm{B}$ contains boundary intervals of the signal sample;

(2) Set the elements in set A larger than the positive threshold IOU as positive sample, and others as negative sample, and discard the remaining samples. Since $B \subset A$, at least one positive sample exists. In this paper, the positive and negative thresholds of this IOU are set as 0.7 and 0.3 respectively.

(3) If the number of positive samples is larger than the set number of positive samples, part of the positive samples will be discarded at random; the same processing is performed on the negative samples.

The training samples of abnormal signal prediction interval generation module are also derivative samples of the model training samples. And their generation process is:

(1)Compute the IOU between the elements in sets A (all the proposal intervals) and B (the boundary intervals of the signal samples themselves);

(2) Through the IOU calculated in Step 1, the corresponding true boundary interval of each proposed interval can be obtained, and the IOU corresponding to the true boundary interval of each element in set A constitutes set $\mathrm{C}$;

(3) By comparing all elements of set $\mathrm{C}$ with the set IOU threshold, the ones larger than the IOU threshold are positive samples, while the others are set as negative samples. If there are too many positive samples, some of them will be discarded randomly, thus generating the label of positive and negative samples. Set the IOU threshold used here to 0.5 in this paper;

(4) Calculate the position transformation parameters of the real boundary interval corresponding to the sample of the generated label.

\subsection{Model training}

The principle of labeling the boundary of the local abnormal signal in a signal is: the mean value of a signal is taken as the dividing line, the position of the abnormal signal 
that begins to cross dividing line as the starting point, and the position when it crosses the dividing line as the end point. Since the signal is not filtered, there may be some signals that fluctuate near the dividing line.

In the model training, the loss function of the proposal interval generation module is the sum of the losses of the classification and regression models, as shown in Eq. (6).

$$
L_{r p n}\left(\left\{p_{i}\right\},\left\{t_{i}\right\}\right)=\frac{1}{N_{c l s}} \sum_{i} L_{c l s}\left(p_{i}, p_{i}^{*}\right)+\frac{1}{N_{r e g}} \sum_{i} p_{i}^{*} L_{r e g}\left(t_{i}, t_{i}^{*}\right)
$$

Where $i$ is the index of the proposal interval, $p_{i}$ is the probability that the $i^{\text {th }}$ sample is predicted as an abnormal signal, $p_{i}^{*}$ is the true label corresponding to the $i^{\text {th }}$ sample, and $t_{i}=\left\{t_{x}, t_{w}\right\}$ are two prediction regression interval transformation parameters, $t_{i}^{*}$ are the transformation parameters of two proposal interval of positive samples, $L_{c l s}$ is the Cross-Entropy loss function used in the classification model, $L_{r e g}$ is the Smooth L1 loss function used in the regression model, $N_{c l s}$ is the number of classification samples, and $N_{\text {reg }}$ is the regression samples number. In this article, $N_{c l s}$ is 256 , and $N_{\text {reg }}$ is 200 .

The loss function of the abnormal signal prediction interval generation module is also represented in Eq. (6). The difference is that $N_{c l s}$ is 128 and $N_{\text {reg }}$ is 128 .

The loss function of 1D-Faster R-CNN is the sum of the loss function of proposal interval generation module and abnormal signal prediction interval generation module, which is shown in Eq. (7).

$$
L_{1 D-\text { faster }-r c n n}=L_{r p n}+L_{r o i}
$$

In this paper, Adaptive moment estimation (Adam) algorithm [18], whose two super parameters are set as follows: $\beta_{1}=0.9, \beta_{2}=0.999$, is used to train the model. Set the initial learning rate to 0.0001 . During the training process, each batch contains one sample, and 1000 iterations of training are performed. After each iteration of training, calculated the average precision of the test dataset. The loss curve and the average precision curve of the training process of a certain batch of samples are shown in Figure 4.

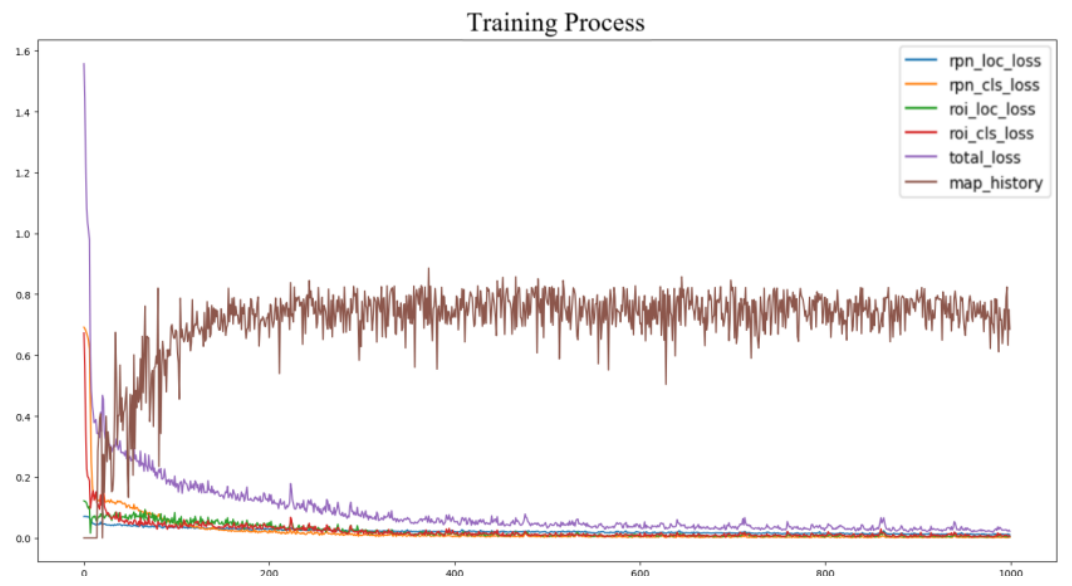

Figure 4. Training process. 
It can be seen from the Figure 3 that the model tends to be stable at about the 500th iteration, and the training model, when the best average precision value is saved during the training process, is 0.877 at this time. Figure 5 shows the extraction effect of abnormal signals from leak signals.
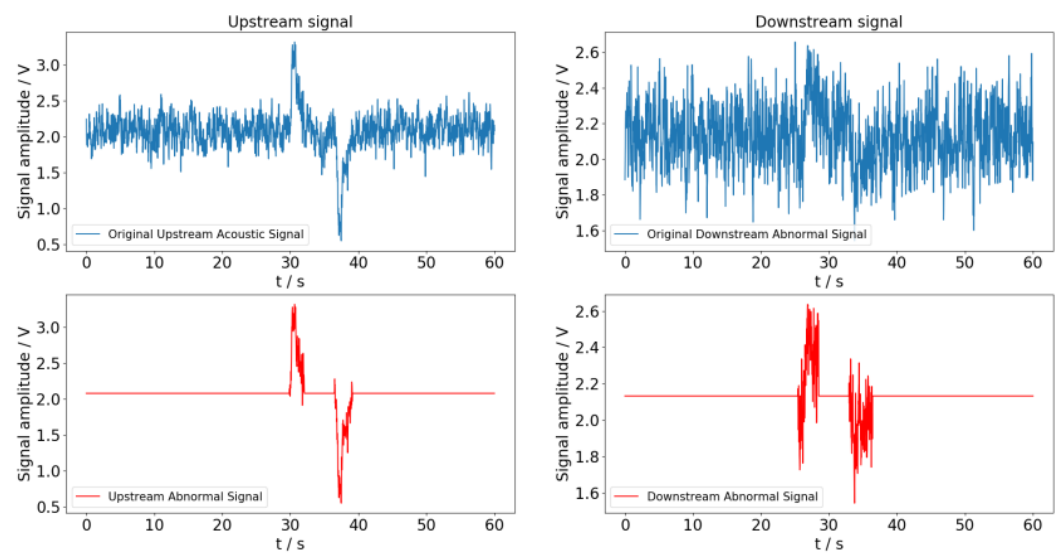

Figure 5. Extraction effect of abnormal signals from leak signals.

\section{Validity test of method}

The effectiveness of the abnormal signal detection method proposed in this paper is verified experimentally with a naphtha transmission pipeline as the experimental platform. The pipeline parameters and operating conditions are the same as literature [14]. Acoustic signal transmitters are installed in the upstream and downstream stations and the acoustic signal is collected synchronously. Among them, on November 20 and 21,2013 , a total of 15 burst leak simulation experiments were carried out on the naphtha transmission pipeline at a distance of $9.476 \mathrm{~km}$ from the upstream.

\subsection{Modeling}

Two leak signals, four station operation signals and four random interference signals are selected as the training dataset, and 10 samples are randomly selected from the known signals containing local abnormal signals as the test dataset. Using the method in Section 2.5 for modeling, the average precision of the upstream and downstream models on their respective verification datasets is 0.82 and 0.87 , respectively. Combined with the cross-correlation localization method based on signal matching in literature [14], it is used as a complete leak detection and localization algorithm.

\subsection{Comparative experiment}

In the historical data collected from November 3, 2013 to December 17, 2013 of the naphtha pipeline, there were a total of 53,006 valid signal samples, and the above signal samples are used as diagnostic objects. This paper compares the local abnormal signal extraction algorithm with the model-free abnormal signal extraction algorithm 
proposed in literature [14] from four aspects: false alarming, missing alarming, location errors and average sample detection time. The method in literature [14] is implemented by Matlab2019b and the proposed method is implemented by python3.6.5 and pytorch1.2. Compare from the perspectives of false alarm, missing alarm, location error and average sample detection time. The hardware platform required for the algorithm to run is shown in Table 1, and Table 2 shows the comparison results.

From the experimental results in Table 2, it can be seen that both methods have not missing alarm, but both caused false alarms. False alarms of the abnormal signal extraction method of 1D-Faster R-CNN are mainly the operation of the stations that cause false alarms due to inaccurate location ( 3 false alarms), and non-homologous interference signals (11 false alarms). In addition to the above two reasons, there are 16 false alarms that diagnose normal signals as abnormal signals. From the perspective of abnormal signal recognition and real-time, the proposed method in this paper has high accuracy and fast calculation speed, which meets the requirements of practical applications.

Table 1. Related parameters of hardware experiment platform

\begin{tabular}{ll}
\hline Parameters & Value/Unit \\
\hline Memory & $8 \mathrm{G}$ \\
Video Memory & $6 \mathrm{G}$ \\
CPU & Intel Core i7-9750H \\
GPU & NVIDIA RTX 2060 \\
\hline
\end{tabular}

Table 2. Comparison results

\begin{tabular}{lllll}
\hline \multirow{2}{*}{ Method } & \multicolumn{2}{l}{ Number of Alarms } & \multirow{2}{*}{ Largest Location Error(m) } & \multirow{2}{*}{ Average detection time(s) } \\
\cline { 2 - 4 } & False & Missing & & 0.032 \\
\hline This paper & $\mathbf{1 4}(\mathbf{0 . 0 2 6} \%)$ & $\mathbf{0}(0 \%)$ & $167(1.08 \%)$ & $\mathbf{0 . 0 1 7}$ \\
Model-free & $23(0.043 \%)$ & $\mathbf{0}(\mathbf{0} \%)$ & $\mathbf{8 5}(\mathbf{0 . 5 4 9 6 \% )}$ & \\
\hline
\end{tabular}

\subsection{Experiment on the effect of local abnormal signal extraction}

Construct the dataset to compare the extraction effects of the method in this paper and the method of model-free abnormal signal extraction. When constructing the dataset, in order to eliminate the subjective factors of artificially judging whether the signal is abnormal, the following principles are used to construct the dataset:

- Select the signal frame containing obvious local abnormal and noncontroversial parts;

- Select the signal frame with known operation;

- Select the signal frame where there is obviously no local abnormal signal;

- Record continuous outliers in a frame signal as one local abnormal signal.

According to the above four principles, 400 signal frames are selected to form the dataset. Among them, there are 200 signal frames containing local abnormal signals and 200 signal frames containing no local abnormal signals. A total of 290 local abnormal signals are included.

Compare the two methods from the perspectives of the accuracy of local abnormal signal detection and the accuracy of whether the signal frame contains local abnormal signals. The experimental results are shown in Table 3.

The experimental results show that the proposed method is superior to the comparison method in terms of the detection accuracy of local abnormal signals and whether the signal frame contains local abnormal signals. Moreover, the accuracy of 
the constructed dataset can reach more than $95 \%$, so the method proposed in this paper is effective in the aspect of local abnormal signal extraction.

Table 3. Experimental results of local abnormal signal extraction

\begin{tabular}{lll}
\hline Method & Accuracy of local abnormal signal detection & Classification accuracy \\
\hline This paper & $\mathbf{9 5 . 2 \% ( 2 7 6 / 2 9 0 )}$ & $\mathbf{9 9 . 5 \% ( 3 9 8 / 4 0 0 )}$ \\
Model-free & $75.2 \%(218 / 290)$ & $81.8 \%(327 / 400)$ \\
\hline
\end{tabular}

\section{Conclusion}

This paper proposes a local abnormal signal extraction method for pipeline leak detection based on 1D-faster R-CNN. It can detect local abnormal signals of different amplitude, shape and time-frequency domain characteristics by the application of target detection to one-dimensional signal processing. From the perspective of signal preprocessing, this paper only highlights the abnormal part of the signal through zscore standardization. While avoiding the influence of filtering boundary effect on the signal in the traditional method, it also realizes the detection of small leak. In terms of the model, this paper proposes a method for generating candidate intervals related to anchors for one-dimensional signals, which effectively realizes the coverage of multiscale candidate intervals on the whole frame signal. In addition, based on the characteristics of the derived samples in 1D-Faster R-CNN, it overcomes the problem of fewer leaking samples in the actual pipeline, and realizes the modeling of small samples, which reduces the dependence of the modeling on a large number of samples. Finally, the combination of the abnormal signal extraction method and the crosscorrelation location method based on signal matching in this paper realizes a new type pipeline leak real-time monitoring method, which can effectively reduce false alarms while achieving accurate detection of pipeline leaks.

\section{References}

[1]. Lu Z, She Y, Loewen M.. A Sensitivity Analysis of a Computer Model-Based Leak Detection System for Oil Pipelines. Energies, 2017, 10, 1226.

[2]. He G, Liang Y, Li Y, Wu M, Sun L, Xie C, et al.. A method for simulating the entire leaking process and calculating the liquid leakage volume of a damaged pressurized pipeline. Journal of Hazardous Materials, 2017, 332: 19-32.

[3]. Wang X, Ghidaoui M. Processing S.. Identification of multiple leaks in pipeline: Linearized model, maximum likelihood, and super-resolution localization. Mechanical Systems and Signal Processing, $2018,107$.

[4]. Qu Z, Wang Y, Yue H, An Y, Wu L, Zhou W, et al.. Study on the natural gas pipeline safety monitoring technique and the time-frequency signal analysis method. Journal of Loss Prevention in the Process Industries, 2017, 47.

[5]. Lang x, Li P, Hu Z, Ren H, Li Y.. Leak Detection and Location of Pipelines Based on LMD and Least Squares Twin Support Vector Machine. IEEE Access, 2017, PP:1-1.

[6]. Ranginkaman M, Haghighi A, Mohammad Vali Samani H.. INVERSE FREQUENCY RESPONSE ANALYSIS FOR PIPELINES LEAK DETECTION USING THE PARTICLE SWARM OPTIMIZATION. INTERNATIONAL JOURNAL OF OPTIMIZATION IN CIVIL ENGINEERING, 2016, 6:1-12.

[7]. Wang C, Zhang Y, Song J, Liu Q, Dong H.. A novel optimized SVM algorithm based on PSO with saturation and mixed time-delays for classification of oil pipeline leak detection. Systems Science \& Control Engineering, 2019, 7:75-88.

[8]. Xiao R, Hu Q, Li J. Leak detection of gas pipelines using acoustic signals based on wavelet transform and Support Vector Machine. Measurement, 2019, 146:479-89. 
[9]. Hori T, Watanabe S, Zhang Y, Chan W. Advances in Joint CTC-Attention based End-to-End Speech Recognition with a Deep CNN Encoder and RNN-LM. 2017.

[10]. Kiranyaz S, Ince T, Gabbouj M.. Real-Time Patient-Specific ECG Classification by 1D Convolutional Neural Networks. IEEE transactions on bio-medical engineering, 2015, 63.

[11]. Kang J, Park Y-J, Lee J, Wang S-H, Eom D-S.. Novel Leakage Detection by Ensemble CNN-SVM and Graph-Based Localization in Water Distribution Systems. IEEE Transactions on Industrial Electronics, 2017, PP:1-1.

[12]. Zhou M, Pan Z, Liu Y, Zhang Q, Cai Y, Pan H.. Leak Detection and Location Based on ISLMD and CNN in a Pipeline. IEEE Access, 2019, PP:1-1.

[13]. Wang F, Lin W, Liu Z, Wu S, Qiu X.. Pipeline Leak Detection by Using Time-Domain Statistical Features. IEEE Sensors Journal, 2017, PP:1-1.

[14]. Wang F, Lin W, Liu Z, Qiu X.. Pipeline Leak Detection and Location Based on Model-Free Isolation of Abnormal Acoustic Signals. Energies, 2019, 12:3172.

[15]. Girshick R, Donahue J, Darrell T, Malik J. Rich feature hierarchies for accurate object detection and semantic segmentation. Proceedings of the IEEE Computer Society Conference on Computer Vision and Pattern Recognition, 2013, 2014.81

[16]. Girshick R.. Fast r-cnn. ICCV. 2015, 2015.169

[17]. Ren S, He K, Girshick R, Sun J. Faster R-CNN: Towards Real-Time Object Detection with Region Proposal Networks. IEEE Transactions on Pattern Analysis and Machine Intelligence, 2015,39.

[18]. Kingma D, Ba J., Adam: A Method for Stochastic Optimization. International Conference on Learning Representations. 2014. 